\title{
Der Partitiv als Objektkasus im Finnischen*
}

0. Dem fi. Partitiv ist reichlich Aufmerksamkeit geschenkt worden: Die Distribution der Kasus beim Subjekt (Nominativ/Partitiv), Objekt (Akkusativ/Partitiv) und Prädikativ (Nominativ/Partitiv) dürfte wohl das meisterörterte Problem der fi. Syntax sein. Die Diskussion um das Objekt hat mehrere Etappen durchlaufen, die gekennzeichnet sind durch die Benennungen wie 'total' versus 'partial', die Aspekte 'resultativ' und 'irresultativ' und neuerlich 'markiert' versus 'unmarkiert'. Letztere scheint durch die Feststellung Auli Hakulinens und Fred Karlssons (1979) eingeleitet worden zu sein, daß der Partitiv der üblichste Objektkasus sei. Ihre Schlußfolgerung, schnell verbreitet und akzeptiert, ergibt den Partitiv als "den" Objektkasus ("unmarkiert") und erklärt den Akkusativ als Nicht-Partitiv ("markiert"). Für meinen Ausgangspunkt, den Unterricht für Nicht-Muttersprachler, sind die Probleme dadurch noch nicht aus der Welt. Auch die neue Entwicklung zeigt aus dieser Perspektive das gleiche Handikap wie die bisherigen. Hans Fromm hat es sehr treffend formuliert: Der finnische Linguist, der sowieso weiß, welcher Kasus gewählt werden muß, schließe aus dem Kasus auf die Art der Satzaussage, rekurriere also von syntaktischer Struktur nach semantischem Inhalt, während die Regelfassung den umgekehrten Weg gehe (Fromm 1982:172 Anm.). Mit anderen Worten: Aufgrund dieses Wissens gibt er dem Verb das Prädikat 'irresultativ' oder 'resultativ' oder, in neuerer Fassung, entscheidet, welcher Kasus 'unmarkiert' vorkommt. Die Beschreibung als 'Aspekt' hat wiederum den Nachteil, daß man mit 'Aspekt' in den verschiedenen Spra-

* Dieser Artikel ist eine umgearbeitete Fassung meines Vortrages, gehalten auf dem VI. Finnougristen-Kongreß in Syktyvkar, am 29. Juli 1985. Ich danke hiermit allen Diskussionsteilnehmern, besonders Herrn Dr. Aleksander Volodin, für Kritik und Anregung. 
chen auf ganz unterschiedliche Systeme zu verweisen scheint (vgl. z.B. De Groot - Tommola 1984). Eeva Kangasmaa-Minn hat die strukturellen Unterschiede $z$ wischen dem $\mathrm{Fi}$. und den indogermanischen Sprachen zutreffend hervorhoben (Kangasmaa-Minn ibid. 1984).

Aus kontrastiver Perspektive drängen sich auch andere Fragen auf. Warum sind Finnen mit Schwedisch als Muttersprache an ihren Objektfehlern zu erkennen? Gerade über den Partitiv stolpert auch derjenige, der sonst die $Z$ weitsprache perfekt beherrscht. Die Objektregeln sind also diejenigen, die am nachhaltigsten Schwierigkeiten verursachen. Auf der anderen Seite ist der Verfall der Sprache bei Auswanderen und ihren Kindern zuerst an u.a. Objektfehlern zu beobachten (vgl. Erling Wande 1985). Das Kasussystem beim Objekt ist also leicht zu beeinträchtigen.

Von diesen Beobachtungen her stelle ich die Frage, ob es nicht nützlich wäre, die Objektregeln in einem anderen strukturellen Rahmen zu betrachten. Man kann annehmen, daß es einen strukturellen Unterschied zwischen dem fi. und den indogermanischen Objektsystemen gibt; die Schwierigkeiten wären dann auf die Unversöhnlichkeit dieser Systeme zurückzuführen. Meine Hypothese lautet, daß der Partitiv und Akkusativ im Fi. nicht grammatisch oder syntaktisch, sondern semantisch sind, während der Akkusativ in den indogermanischen Sprachen sich zu einem grammatischen Kasus entwickelt hat. Meine Ausführungen sind insofern spekulativ, als die Belege aus dem heutigen Sprachbestand kommen; das heißt, daß eine diachrone Entwicklung von den heutigen Erscheinungen her postuliert wird.

Die theoretische Begründung kommt aus der sog. lokalistischen Hypothese. In dieser Theorie werden die komplizierten semantischen und syntaktischen Beziehungen auf eine primäre kognitive Basis der räumlichen Orientierung zurückgeführt; einen guten Úberblick bietet u.a. Lyons (1977:718724). Nach einer Übersicht über das fi. Kasussystem (1) bespreche ich die Funktionen des Partitivs von einer "ablativen" Proto-Bedeutung aus. Auch der Akkusativ erhält einen lokalistischen Hintergrund (2). Im dritten Abschnitt wird die tatsächliche Distribution des Partitivs und Akkusativs durch eine Einteilung in vier Kategorien beschrieben. Zuletzt erörtere ich einige Probleme, die sich aus dieser Verteilung ergeben. 


\section{Das finnische Kasussystem}

1.1. Betrachten wir die Lokative, so scheint das fi. Kasussystem gut entwickelt, folgerichtig und kohärent zu sein. Es gibt dreimal drei Kasus, die die Orientierung im Raum ausführlich beschreiben und festlegen. Die Tripartition zeigt ein System mit statischen Kasus für das Sich-Befinden (drei "Essive") und dynamischen für die Bewegung (drei "Ab-Lative" und drei "Ad-Lative"). Die Orientierung innerhalb eines dreidimensionalen Raumes wird durch die inneren Lokative oder -s-Kasus Inessiv, Elativ und Illativ beschrieben, z.B. huonee-ssa 'im Zimmer', huonee-sta 'aus dem Zimmer' und huonee-seen 'in das Zimmer' (der Illativ zeigt Allomorphe, hier nicht zu berücksichtigen). Die äußeren Lokalkasus Adessiv, Ablativ und Allativ haben als gemeinsames Zeichen -1 - und verweisen auf einen zweidimensionalen Raum, z.B. tie-llä 'auf dem Wege', tie-ltä 'vom Wege' und tie-lle 'auf den Weg'. Diese Lokative gehen auf die älteren Lokative zurück; der Essiv mit $\operatorname{dem}$ Suffix $-n A$ ist noch im Inessiv und Adessiv sichtbar $(s+n A>\operatorname{ss} A ; \ln \cap>$ Il $A$ ) und der Partitiv - $t A$ (heutzutage Allomorphe $-t A$ und $-A$ ) im Elativ und Ablativ $(s+t A, l+t A)$.

Die älteren Lokalkasus funktionieren nun eindimensional. Diese Dimension heißt 'Dauer': sie bezeichnen 'Zeit' oder 'Sich-befinden innerhalb eines Zustandes' unter drei verschiedenen Aspekten. Der Essiv beschreibt einen Zustand als eine Ganzheit:

la Lapsena hän oli usein sairas. Als Kind war er oft krank.

Der Partitiv bezeichnet "Bewegung innerhalb des Raumes":

lb Hän tulee tätä tietä. Er kommt diesen Weg entlang.

Der Translativ verweist auf eine Veränderung des Zustandes oder auf eine Bewegung, die die Grenze des Raumes überschreiten wird:

lc Hän maalaa oven mustaksi. Er streicht die Tür schwarz (an). 
Die Kasus bezeichnen einen temporalen Raum (z.B. joulu 'Weihnachten') von verschiedenen Aspekten her:

2 Hän on täällä jouluna.

Er ist hier an Weihnachten.

(Essiv: globale Zeitangabe)

joulua. Er ist hier zu Weihnachten.

(Partitiv: um Weihnachten zu feiern)

jouluksi. $\quad$ Er ist hier für Weihnachten.

(Translativ: kommt hierher für Weihnachten

/kommt vor Weihnachten und bleibt dann.)

1.2. Verglichen mit diesem kompakten System sind die grammatischen oder syntaktischen Kasus im Finnischen schwach entwickelt, nicht folgerichtig und inkohärent. 'Schwach entwickelt' heißt, daß es keinen Kasus gibt, der eindeutig die Satzglieder markieren würde: 'Nominativ' und 'Partitiv' erscheinen als Kasus für Subjekt, Objekt und Prädikativ. 'Nicht folgerichtig' heißt, daß es Allomorphe für den Objektkasus im Singular gibt $(-n,-t$ und 0$)$ und keine Distinktion im Plural (das Objekt erscheint im Nominativ oder Partitiv). Der Kasus des Objektes ist vom Satzbau abhängig: ohne ein Nominativsubjekt wird das Objekt anders markiert als mit einem solchen, und für einen negativen Satz gelten eigene Regeln. 'Inkohärent' heißt, daß es viele Konstruktionen gibt, wo eine NP in einem Lokalkasus die Funktionen eines 'Subjekts', 'Objekts' und 'Prädikativs' übernimmt: man denke nur an die Konstruktionen für 'haben' und 'müssen' mit "Adessiv-" und "Genitivsubjekten".

1.3. Es fällt auf, daß das Element $-n$ vielseitig verwendet wird: es ist das Suffix für den Genitiv, der ja u.a. im dativischer Bedeutung verwendet wird, aber auch formgleich ist mit dem Akkusativ Sg; formgleich war auch der Instruktiv $\mathrm{Sg}$, nunmehr praktisch verschwunden. Das $-n$ bildet einen festen Teil der Illativendungen und erscheint ebenfalls im Komitativ (-ne). Spekulativ können wir fragen, ob man nicht eine semantische Konnotation für dieses Element annehmen könnte. Diese Kasus haben nämlich einen gemeinsamen Faktor in ihrer Bedeutung: man könnte ihn als "Verbindung oder Berührung von zwei Entitäten" bezeichnen. Diese Annahme ist rein spekulativ; wenn man aber dem Element $-s$ bei inneren und $-l$ bei äußeren Lokalkasus eine 
semantische Konnotation beimißt, kann man sie keineswegs für ganz unmöglich halten.

\section{Partitiv als Ablativ}

2.1. Die Proto-Bedeutung des Partitivs dürfte wohl ein Ablativ sein, die Bewegung von einem Ausgangspunkt bezeichnend, also 'ab', 'aus' oder 'von'. In der Bedeutung "fortschreitende Bewegung" wird der Partitiv noch heute sowohl in einem konkreten (Beispiel 1b) als abstrakten (Beispiel 2) Sinn verwendet. Im System der räumlichen Orientierung könnte man ihm die Dimension 'entlang' geben, wie z.B. in

3 Kärpänen marssii pöytää. Die Fliege marschiert den Tisch entlang.

Die Vorstellung "fortschreitende Bewegung" scheint kognitiv primär zu sein; dem finnischen "olemme yhtä mieltä" mit einem Partitiv-Ablativ entspricht wohl lateinisch "uno sumus animo". Die Proto-Bedeutung manifestiert sich auch in 'Subjekt' und 'Prädikativ', die jetzt nicht berücksichtigt werden können, die aber organisch hier zusammengehören. Wir konzentrieren uns in diesem Zusammenhang auf die Frage: Warum ist der Partitiv im Finnischen ein "Objektkasus"? Folgende Beispiele illustrieren die Erweiterung der räumlichen Perspektive:

- Bewegung als fortdauernde Art und Weise:

4a Hän ui kroolia. Er schwimmt Kraul.

4b Hän laulaa toista ääntä. Er singt die zweite Stimme.

- Ausgangspunkt für einen Zustand:

5a Katu kiiltää märkyyttään. Die Strasse glitzert vor Nässe.

5b Talo kuhisee väkeä. Das haus wimmelt von Leuten.

- Zustand, determiniert durch einen entscheidenden Punkt:

6a Tulemme sitä kautta. Wir kommen dort entlang.

6b Olemme sitä kautta. Wir sind aus jener Zeit.

/Wir gehören zu der Zeit.

6c Elämme sitä kautta. Wir erleben jene Zeit.

/Wir leben in der Zeit. 
- Ursache von einer Bewegung oder einem Zustand:

7a Hän pakenee minua. Er flieht vor mir.

7b Hän pelkää minua. $\quad$ Er hat Angst vor mir.

7c Hän inhoaa minua. Er verabscheut mich.

2.2. Bei der Entwicklung des Partitivs dürfte die Ausbildung des Elativs auch eine Rolle gespielt haben. Eine Synonymität erscheint nunmehr nur in einigen Adverben, wo der Partitiv eine konkrete lokative Bedeutung aufweist:

8a Hän soittaa kotoa. Er ruft von zu Hause an.

8b Hän soittaa hotellista. Er ruft aus dem Hotel an.

Die Entwicklung scheint dazu geführt zu haben, daß der Elativ den konkreten Ausgangspunkt für sich monopolisiert hat, während im Bereich der abstrakten Bedeutung eine Arbeitsteilung entstanden ist. Vergleiche

9a Hän puhuu hotellista. $\quad$ 1. Er spricht vom Hotel aus.

2. Er spricht über das Hotel.

9b Hän puhuu suomea. $\quad$ Er spricht Finnisch.

$9 c$ Hän soittaa viulua. Er spielt Violine.

Vergleiche auch folgende Minimalpaare:

10a Se on tammea.

tammesta.

lob Se on Mozartia.

Mozartista.

10C Se on Helsinkiä.

Helsingistä.

10d Kaksi poikaa lähtee.

pojista
Es ist Eiche. aus Eiche.

Es ist Mozart (z.B. ein Stück Musik). über Mozart.

Es ist Helsinki (z.B. ein Bild; Stadt teil). aus Helsinki.

Zwei Jungen gehen. von den Jungen gehen.

(Vergleiche z.B. Pentti Leino 1982:13-14) 
2.3. Eine theoretisch interessante Tatsache ist, daß der Partitiv auch in Opposition steht zum entgegengesetzten dynamischen Kasus, dem Illativ. Die Erklärung dafür dürfte von der Proto-Bedeutung des Partitivs als "fortschreitender Bewegung innerhalb eines Raumes" abzuleiten sein: man kann sie ebensogut interpretieren als "von einem Punkt ausgehende und gegen ein Endziel fortschreitende Bewegung". Analog zum Elativ hat der Illativ für sich die Bezeichnung des konkreten (definiten) Endpunktes monopolisiert, wogegen der Partitiv allgemeiner eine "Bewegung in Richtung auf einen Endpunkt" bezeichnet. Das erklärt, warum der Partitiv und Illativ mal nahezu synonym sind, mal eine deutlichen Unterschied markieren (vgl. zu dieser Frage die Beobachtungen von Paavo Siro 1956 und z.B. Matti Leiwo 1983).

lla Hän lyö palloa. $\quad$ 1. Er spielt Ball. 2. Er schlägt den Ball.

1 lb Hän lyö palloon. $\quad$ Er schlägt gegen den Ball.

12a Hän katsoo minua. Er sieht mich an (= eine Zeitlang)

12 b Hän katsoo minuun. Er blickt mich an (= richtet den Blick auf mich)

13a Hän uskoo minua. Er glaubt mir (, was ich sage).

13b Hän uskoo minuun. Er glaubt an mich (, was ich werde).

Der Illativ bezeichnet das "Objekt" der Handlung bei Verba wie z.B. tutustua 'kennenlernen' väsyä 'müde werden' oder rakastua 'sich verlieben'. Es gibt aber Verba mit dem gleichen reflexiven Suffix -UA, die den Partitiv regieren, wie z.B.

14b Hän pelästyi minua. Er erschrak vor mir.

14b Hän hämmästyi minua. Er wunderte sich über mich.

Semantisch drückt hier der Partitiv die "Ursache" für die Handlung aus, während ein Verb wie tutustua 'kennenlernen' mit dem Illativ die Richtung oder das Endziel der Handlung bezeichnet. Im folgenden Fall bezeichnet der Illativ eine direkte Berührung, während der Partitiv die "Ursache" der Handlung angibt: 
15a Hän kompastui kiveen. Er stolperte über den Stein.

15b Hän varoo kiveä. Er versucht den Stein zu vermeiden.

(Er nimmt sich vor dem Stein in acht.)

Die gleiche Opposition läßt sich im folgenden Paar unterscheiden:

16a Puu puhkeaa lehteen. Der Baum schlägt aus.

(Wörtl.: Der Baum bricht ins Blatt.)

16b Puu tulvii kukkia. Der Baum ist voller Blüten.

(Wörtl.: Der Baum flutet Blumen.)

2.4. Wollte man diese Ausführungen schematisch wiedergeben, könnte man die Proto-Bedeutung des Partitivs durch die folgende (grobe) Skizze veranschaulichen, wo die implizite Bedeutung mit einer-abgebrochenen und die direkte mit einer unabgebrochenen Linie wiedergegeben wird:

Figur 1

Partitiv

$X \longrightarrow \ldots . . .-. .-Y$

Die Lokalkasus Elativ und Illativ beziehen sich auf den Ausgangs- bzw. Endpunkt der Handlung innerhalb des Raumes:

Figur 2

$\mathrm{X} \longrightarrow \mathrm{Y}$

Elativ Illativ

Meine Hypothese lautet nun, daß wegen der gut entwickelten, symmetrischen und folgerichtigen Lokalbezeichnung auch das abstrakte System eine Präzisierung benötigte. Der Partitiv hatte, weil sich die Oppostion zu den Lokalkasus auf konkrete Fälle hin entwickelte, eine allgemeinere Opposition nötig, die man folgenderweise beschreiben kann:

Figur 3

$X-\ldots . . .-. \longrightarrow Y$ 
Der "Akkusativ" dürfte innerhalb der Lokalbezeichnungen entstanden sein und sich von einer Bezeichnung der 'Bewegung in die Richtung des Endzieles' entwickelt haben. Es gab schon das Element $-n$ mit einer Konnotation "direkte Berührung zweier Entitäten", wie oben (1.3.) postuliert wird. Ich lasse die Möglichkeit eines Elements - $m$ offen; auch diese Endung dürfte eine begrenzte und vielleicht diffuse Bedeutung gehabt haben. Der "Akkusativ" könnte sich gleichzeitig mit dem Translativ entwickelt haben; die dynamischen Kasus und die deutliche Systematisierung der inneren und äußeren Lokalkasus müssen eine Umordnung aller früheren Kasusbedeutungen verursacht haben. Da der Illativ und Allativ ebenfalls das "Erreichen des Zieles" bezeichneten, hat der Translativ eine andere, und zwar die Bedeutung "am Endpunkt vorbei" entwickelt. Da der Illativ das "Erreichen des Zieles" bezeichnete und der Partitiv die "Bewegung in die Richtung des Zieles; Ursache der Bewegung" andeutete, hat der Akkusativ etwas anderes bedeuten müssen.

Spekulativ können wir annehmen, daß der Akkusativ eine Lücke im System der Lokalbezeichnung hat füllen müssen. Er bezeichnet eine innerhalb eines Raumes entstandene Bewegung, die nicht mehr weitergeht, wenn das Endziel erreicht ist und durch das Erreichen des Zieles terminiert wird. Er impliziert auch, daß die Bewegung vom Agens ausgeht, nicht von einem "Ausgangspunkt" oder einer "Ursache" ausgelöst wird.

Mit diesen Ausführungen habe ich versucht zu zeigen, daß man den Akkusativ auch von dem typisch fi. System der Lokalbezeichnung her erklären kann. Man braucht ihn keineswegs nur für einen "grammatischen" Kasus zu halten, der entstanden sei, um ein 'Subjekt' vom 'Objekt' zu unterscheiden. Man könnte auch fragen, ob es vielleicht einen semantischen Grund für die Regel gäbe, daß nur die Anwesenheit eines Nominativsubjekts die explizite Markierung des Objekts auslöst. Auch diese Sachlage läßt sich semantisch und von der lokalistischen Begründung her erklären. Der Imperativ, zum Beispiel ota kirja! 'Nimm das Buch!' bezeichnet eine Situation, die offen liegt: man weiß noch nicht, in welche Richtung sie sich entwickeln wird, und daher verwendet man das $-n$ nicht. Ein Satz wie Otan kirjan 'Ich nehme das Buch' bezeichnet eine Situation, die terminiert wird. Könnte man also für alle Gebrauchsfälle des Partitivs bzw. Akkusativs einen gemein- 
samen Nenner oder einen invarianten semantischen Inhalt annehmen? Diese Frage wird im folgenden Abschnitt behandelt.

\section{Vier Kategorien}

3.1. Die Distribution der Objektkasus geschieht im Finnischen in vier möglichen Kategorien, in zwei Hauptklassen mit einer Unterteilung in der letzteren:

I Objekt ausschließlich Partitiv

II Objekt Partitiv oder Akkusativ

\section{Objekt ausschließlich Partitiv}

Als gemeinsamen Nenner für Verba in dieser Kategorie (ich gebe nur Beispiele) kann man die Komponente 'Bewegung' finden, wie paeta 'flüchten', seurata 'folgen', totella 'gehorchen', noudattaa 'nachkommen', soveltaa 'anwenden', aber auch Verba für einen "fortschreitenden Zustand" wie odottaa 'warten', pelätä 'fürchten', inhota 'verabscheuen'. Es scheint, daß diese Verba in vielen Sprachen, z.B. im Englischen und Deutschen, Präpositionen aufweisen, wie 'warten auf jemanden'.

Ila Partitivpetale Verba

Folgende Beispiele veranschaulichen eine Erscheinung, die ich mit dem Terminus "Partitivpetal" bezeichnen möchte:

17a Hän lyö palloa.

$17 b$ Hän lyö pallon kentältä.

18a Hän heiluttaa puuta.

$18 \mathrm{~b}$ Hän heiluttaa puun kumoon.

19a Hän kiittää minua.

19b Hän kiittää minut maasta taivaaseen.
Er spielt Ball.

Er schlägt den Ball aus dem Feld.

Er rüttelt an dem Baum.

Er schüttelt den Baum um.

Er dankt mir.

Er lobt mich in allen Tonarten

("von der Erde in den Himmel").

'Partitivpetal' heißt, daß ein Verb den Partitiv regiert, falls kein terminierter Zustand bezeichnet werden soll. Es scheint mir, daß Verba aus dieser 
Kategorie eine Bewegung bezeichnen, ausgehend vom Agens in die Richtung des Objektes, wie z.B. lyödä 'schlagen', heiluttaa 'schwenken', potkia 'mit dem Fuß stoßen', also handlungen, die mit der Hand oder dem Fuß verrichtet werden. Diese Kategorie enthält aber auch Verba wie käyttää 'gebrauchen', puhua 'sprechen' und sovittaa 'anpassen' oder ajatella 'denken' und kuunnella 'zuhören' für kompliziertere Handlungen. In dieser Kategorie bezeichnet der Akkusativ, daß die referierte Handlung ein definites und spezifisches Ziel hat. Man beachte die Ubersetzungen: Diese Sachlage kann im Deutschen durch ein anderes Verb ausgedrückt werden. Im Finnischen bezeichnet der Partitiv also den "gewöhnlichen" Gang der Handlung, der Akkusativ das Ziel dieser Handlung. In dieser Kategorie muß eine Spezifikation explizit ausgedrückt werden durch eine NP in einem dynamischen Kasus, oder ein Adverb, das eine Veränderung des Zustandes anzeigt (z.B. rikki 'kaputt'). Zu beachten ist, daß der Kasus nicht statisch sein darf:

17c *Hän lyö pallon kentällä.

$18 \mathrm{c}$ *Hän heiluttaa puun puutarhassa.

Ilb Partitiv-Akkusativ

Diese Kategorie bildet traditionell den Ausgangspunkt für die Objektregeln:

20a Hän kantaa pakettia. Er trägt ein/das Paket.

20b Hän kantaa paketin. Er wird das Paket tragen/trägt das Paket.

$2 l$ a Hän syö leipää.

Er ißt Brot.

21b Hän syö leivän.

Er ißt das/ein Brot.

In dieser Kategorie kann man sowohl den Partitiv als auch den Akkusativ wählen; der Akkusativ setzt also keine zusätzliche Bestimmung voraus (vgl. Kategorie Ila). Ich würde diese Kategorie nicht für die "ursprüngliche" halten, aber vielleicht für eine späte Entwicklung, wo die Sprache die semantischen Eigenschaften des Partitivs und des Akkusativs produktiv, sprachökonomisch und spielerisch benutzt. Der Akkusativ bezeichnet eine Handlung, die innerhalb des referierten Raumes spatiell, temporal oder in einer anderen Weise definit oder terminiert ist, der Partitiv hingegen, daß diese 
Handlung innerhalb des referierten Raumes stattfindet, aber ohne Nachdruck auf ihre Vollendung (vgl. 'bound' versus 'open' Situation, Heinämäki 1984). Für meine Intuition bezeichnet 20b nicht notwendig, daß das Paket zu einem bestimmten Platz gebracht wird - man kann ihn auch so interpretieren, daß die Person etwas anderes nicht tragen wird (will). Satz 2 lb kann aussagen, daß das Brot ganz aufgegessen wird. Er kann aber auch anders interpretiert werden, z.B. daß die Person eine bestimmte Wahl vom Menü trifft, ohne verpflichtet zu sein, das Gewählte gänzlich zu verzehren.

Bisweilen kann aber der Objektkasus die Bedeutung des Verbs verändern:

22a Hän kutsuu minua. $\quad$ Er ruft mich.

22b Hän kutsuu minut. Er lädt mich ein.

Die Vorstellung 'die Stimme erheben' wird hier in zwei verschiedenen Weisen interpretiert, wobei der Partitiv auf die fortdauernde Tätigkeit hinweist und der Akkusativ dieser Tätigkeit ein spezielles Ziel zuschreibt. Die letztere Bedeutung hat sich dann aus der spatiellen Begrenzung befreit, während der Partitiv auf einen konkreten Raum verweist. Vergleiche auch
23a Hän hakee minua.
Er sucht mich.
23b Hän hakee minut.
Er holt mich.

Auch in diesem Fall vermute ich, daß der Partitiv der ursprüngliche Kasus sein dürfte (vgl. z.B. odottaa mit ausschließlichem Partitiv). Sprachökonomisch bedeutet die Systematisierung der Opposition, daß ein Verb ein breites semantisches Feld bestreiten kann. Man kann daher annehmen, daß ein Verb dieser Kategorie über eine breitere Skala Gebrauchsmöglichkeiten verfügt als z.B. partitivpetale Verba.

Zur Frage eines "gemeinsamen Nenners" für diese Kategorie möchte ich mich nicht äußern; tentativ schlage ich einen räumlich indifferenten semantischen Aufbau vor, was die semantischen Komponenten 'Bewegung' und ' $\mathrm{Zu}$ stand' betrifft. Die freie Wahl zwischen dem Partitiv und Akkusativ verweist darauf hin, daß die Handlung von zwei Aspekten her beschrieben werden kann. Es kann aber auch sein, daß diese Kategorie sich als eine Zwi- 
schenzone manifestiert, Verba einschließend, die weder partitiv- noch akkusativpetal sind, und der Sprecher darum von den semantischen Eigenschaften dieser Kasus Gebrauch machen kann.

Es gibt Fälle, wo Partitiv und Akkusativ praktisch dasselbe bedeuten, wie z.B.

$\begin{array}{ll}\text { 24a Hän pyytää minua. } & \text { Er lädt mich ein. } \\ \text { 24b Hän pyytää minut. } & \text { Er lädt mich ein. }\end{array}$

Der praktische Unterschied zwischen diesen Sätzen ist gering, aber sie zeigen die Haltung des Sprechers an. Satz 24 heißt 'Er bittet mich, mitzukommen' (auf ein Fest, ins Kino etc.). Der Akkusativ bezeichnet die Einladung als 'definit', als eine abgemachte Sache. Der Partitiv macht die Einladung 'nicht definit', sie ist keine abgemachte Sache. Satz 24b sagt aus: "ausschließlich mich", Satz 24a läßt auch das offen.

Ilc Akkusativpetale Verba

'Akkusativpetal' heißt, daß ein Verb den Akkusativ regiert, falls es keinen Grund für den Partitiv gibt. Zu akkusativpetalen Verba zähle ich die Bezeichnungen der Aktion eines Sinnesorgans, wie nähdä 'sehen', kuulla 'hören', huomata 'bemerken', oder sanoa 'sagen', ilmoittaa 'melden', väittää 'behaupten' (eine vereinzelte verbale Außerung tun), oder ostaa 'kaufen', omistaa 'besitzen', valita 'wählen', muistaa 'erinnern', taata 'garantieren', (die Handlung setzt ein Objekt voraus), wie auch perustaa 'ergründen' oder tuntea 'kennen IIc; fühlen IIb).

25a Hän näkee minut.

Er sieht mich.

25b Hän maksaa laskun.

Er bezahlt die Rechnung.

25c Hän omistaa talon.

Er besitzt das Haus.

Eine besondere Eigenschaft des fi. Objektsystems besteht darin, daß es keine Kategorie gibt für "ausschließlich Akkusativ". Es gibt nur Verba, die den Partitiv unter bestimmten Umständen nicht zulassen. Auf der anderen Seite gibt es Verba, die den Akkusativ nie zulassen. Die ersteren heißen 
'akkusativpetal'. Es gibt drei Bedingungen, bei denen der Partitiv gewählt werden muß:

1. In der Verneinung stehen alle Objekte im Partitiv.

Die Negation impliziert ja, daß die Verbindung mit dem Ziel der Handlung irrelevant wird. Es ist dabei zu bemerken, daß dieser Zustand verschiedene Interpretationen in der Kategorie IIb zuläßt:
$21 c$ Hän ei syö leipää.
1. Er ißt kein Brot. (vgl. 2la)
2. Er ißt das Brot nicht (vgl. 21b)
23c Hän ei hae minua.
1. Er sucht mich nicht. (vgl. 23a)
2. Er holt mich nicht. (vlg. 23b)

$\mathrm{Zu}$ beachten ist aber, daß in den Kategorien I, Ila und IIc nur eine Interpretation nötig ist.

2. Der Partitiv wird benutzt, wenn die Erreichung des Zieles fragwürdig oder unsicher ist, oder wenn man sie so präsentieren will.
26a Saisinko Liisaa puhelimeen?
Könnte ich Liisa sprechen?
26b Sain Liisan puhelimeen.
Ich konnte Liisa sprechen.
27 a Tunnen häntä vähän.
Ich kenne ith wenig.
$27 \mathrm{~b}$ Tunnen hänet hyvin.
Ich kenne ihn gut.
28a Niitä on vaikea huomata.
Sie sind schwierig zu bemerken.
$28 \mathrm{~b} \mathrm{Ne}$ on helppo huomata.
Sie sind leicht zu bemerken.

3. Der Partitiv muß gewählt werden, wenn es um eine unbestimmte Menge oder indefinite Anzahl geht, da der Akkusativ auf ein "definites" Objekt verweist.
29a Näin verta lattialla.
Ich sah Blut auf dem Fußboden.
$29 b$ Näin veren lattialla.
Ich sah das Blut auf dem Fußboden.
30a Hän omistaa taloja.
Er besitzt Häuser.
$30 \mathrm{~b}$ Hän omistaa talot.
Er besitzt die Häuser.

3.2. Der Unterschied zwischen den Kategorien Ila (partitivpetal) und IIb (Partitiv-Akkusativ) wird syntaktisch ausgedrückt: die Kategorie Ila be- 
nötigt einen zusätzlichen Konstituenten als Bedingung für den Akkusativ, die Kategorie Ilb zeigt Minimalpaare mit drei Satzgliedern. Was ist die Eigenschaft, die die Kategorien IIb und IIc (akkusativpetal) voneinander unterscheidet? Die oben erwähnten Bedingungen ausgenommen: warum läßt die Kategorie Ilc den Partitiv nicht zu?

Mit dieser Frage sind wir beim Kern der Objektproblematik angelangt. Die Schwierigkeiten betreffen also den Kasus für ein Objekt im Singular, das kein Stoffname ist, und das in einem bejahenden Satz vorkommt. Warum sind also in der Kategorie IIb sowohl Partitiv und Akkusativ möglich, in der Kategorie IIC aber nur der Akkusativ? Man vergleiche

31 a Hän lukee kirjaa.

kirjan.

3 lb Hän lukaisee kirjan.

$$
\text { *kirjaa. }
$$

32a * Jalankulkija huomaa takana tulevaa autoa.
Er liest in dem Buch/an dem Buch.

Er liest das Buch aus.

Er liest das Buch schnell durch.

(Der Fußgänger bemerkt

einen von hinten kommenden Wagen)

Das Beispiel 32a stammt von Terho Itkonen (1974(1976):179), der die momentane Art der Handlung als Ursache für die Unmöglichkeit bezeichnet: man kann hier unmöglich ein abtrennbares Moment segmentieren. Diese Erklärung wirkt plausibel: eine Segmentierung macht tatsächlich den Partitiv wenigstens tolerabel, wie z.B.

32b ?Jalankulkija ehti huomata Der Fußgänger konnte den von hinten takaa tulevaa autoa vain kommenden Wagen nur den Bruchteil einer sekunnin murto-osan. Sekunde wahrnehmen.

Die momentane Art der Aktion ist aber nicht der Grund der Akkusativpetalität, um es mit meinem Terminus zu sagen, wie die folgenden Beispiele zeigen:

33a Hän potkaisi palloa. Er trat schnell den Ball.

(Er gab dem Ball einen kräftigen Fußtritt.) 
33b Hän huomasi jotakin

Er bemerkte etwas Blinkendes vor sich.

kiiltävää edessään.

Im Beispiel 33a ist das Verb partitivpetal (siehe potkia la), und es gibt keine Bestimmung, die einen Akkusativ verlangt (z.B. kentältä 'aus dem Feld; rikki 'kaputt'). Im Beispiel $36 \mathrm{~b}$ bezeichnet der Partitiv das Objekt als 'unbestimmt' (Bedingung 3 oben). Im Beispiel 35a scheint es also etwas anderes zu sein, was den Partitiv unmöglich macht.

Wir können auch zugleich eine andere Schwierigkeit besprechen: warum erlaubt das Verb nähdä 'sehen' den Partitiv Plural (Bedingung 3), huomata 'bemerken' aber nicht?

34a Hän näki autoja.

Er sah Autos.

34b *Hän huomasi autoja.

(Er bemerkte Autos.)

Eine räumliche Spezifikation macht den Partitiv tolerabel (ein Fragezeichen bedeutet, daß der Satz nach meiner Intuition "tolerabel", also marginal ist):

34c ?Hän huomasi autoja pihalla. Er bemerkte Autos auf dem Hof.

34d ?Hän huomasi vieraita autoja $\mathrm{Er}$ bemerkte fremde Autos oven edessä. vor der Tür.

34e Hän huomasi vieraat autot oven edessä. Er bemerkte die fremden Autos vor der Tür.

Zwei Bedeutungen sind m.E. zu unterscheiden: 1) die Bedeutung eines Verbs, das semantische Feld der dadurch bezeichneten Aktion, auch als "Aktionsart" bezeichnet, und 2) die Bedeutung der Kasusendung. Daraus ergibt sich die Möglichkeit, daß ein Verb nur bestimmte Kasus zuläßt; einer dieser Fälle heißt 'Akkusativpetalität'. Das Verb huomata dürfte semantische Komponenten haben wie 'imstande sein, etwas durch ein Sinnesorgan wahrzunehmen', 'eine Beobachtung durch ein Sinnesorgan verrichten', 'dem Beobachteten eine Bedeutung geben'. Es scheint stark akkusativpetal zu sein. Meiner Intuition nach ist die letzte Komponente ausschlaggebend: "eine Bedeutung geben" kann nicht als eine "fortschreitende Bewegung" bezeichnet werden. - 
Die erste Komponente kommt in Frage, wenn kein nominales Objekt vorhanden ist. - Statt einer "momentanen Aktionsart" (Terho Itkonen) als Ursache für die Unmöglichkeit eines Partitivobjekts präsentiere ich "eine semantische Zusammensetzung, die keine 'Bewegung' (in konkretem oder abstraktem Sinne) einschließt". Solche Verba heißen 'stark akkusativpetal'; ein Verb wie nähdä ist 'akkusativpetal'.

Das Objekt der Wahrnehmung beim Verb huomata ist 'definit' wegen dieser kognitiven Komponente, was auch bei einer "unbestimmten Venge" die "definite Form" voraussetzt (Hän huomasi veden / * Hän huomasi vettä). Im Falle des Plurals erscheint ein interessantes Dilemma. Die Nominativform 'Hän huomasi autot' ist ohne weiteres akzeptabel, setzt aber 'autot' als bekannt voraus. Der Partitiv Plural 'autoja' wird abgelehnt, trägt aber eine Bedeutung ("indefinite Anzahl"), die nur dadurch ausgedrückt werden kann. Eine räumliche Zufügung macht den Partitiv jedenfalls tolerabel (34c), da sie eine distributive Interpretierung zuläßt ("er bemerkte das Autol + Auto2 + Auto3 ..."), also als eine Serie Wahrnehmungen innerhalb des explizit gegebenen Raumes.

Meiner Intuition nach klingt ein Satz wie im Beispiel 34c jedoch weniger natürlich als 'Hän näki autoja pihalla', der durchaus akzeptabel ist. Das dürfte bedeuten, daß nähdä von einer räumlichen Perspektive her interpretiert werden kann ("visuelle Wahrnehmung verrichten; dauernd damit beschäftigt sein"). Satz $34 \mathrm{~d}$ ist nach meiner Intuition etwas akzeptabler als Satz 34c; das Attribut 'fremd' dürfte ihm eine zusätzliche Legitimierung geben, da sie Rekognition voraussetzt ("er sah, daß die Autos nicht bekannt waren").

Wenn wir die Opposition Partitiv/Akkusativ betrachten, scheint der Akkusativ die Aktion nicht auf eine "Bewegung" zu beziehen, aber auf ihre Vollziehung: der Akkusativ ist "inhärent terminativ". Die "Bewegung" durch den Partitiv kann dahingehend interpretiert werden, daß die Handlung von einem Punkt beginnt und zu einem anderen läuft, oder noch nicht beendet ist, oder fragwürdig ist oder unsicher hinsichtlich das Resultats usw. Der Partitiv ist "inhärent durativ".

3.3. Daraus ergibt sich, daß die spatialen und temporalen Bestimmungen im Einklang damit sein müssen; wir betrachten ein Beispiel, die Kasusdistri- 
bution innerhalb eines temporal begrenzten Rahmens. Die Handlung wird auf zwei Aspekte bezogen. Die Zeitangabe "eine Stunde" segmentiert die Handlung ("x tut y eine Stunde"); die Zeitangabe "in einer Stunde" sagt aus, was man in einer abgeschlossenen (oder abzuschließenden) Episode tut. Wir können daher für den ersteren Fall den Partitiv und für den zweiten den Akkusativ erwarten. Die Beispiele sind nach den obigen Kategorien gruppiert.

\section{- Partitiv}

35a Hän seuraa minua tunnin. Er folgt mir eine Stunde.

35b *Hän seuraa minut tunnissa.

- Partitivpetal

36a Hän lyö palloa tunnin.

36b Hän lyö pallon hetkessä kentältä.

37a Hän heiluttaa puuta tunnin.

38b Hän heiluttaa puun tunnissa kumoon.

- Partitiv-Akkusativ

38a Hän lukee kirjaa tunnin.

38b Hän lukee kirjan tunnissa.

- Akkusativpetal

39a Hän valitsee kirjaa tunnin.

$\mathrm{Er}$ ist eine Stunde mit der Auswahl des Buches beschäftigt.

39b Hän valitsee kirjan tunnissa. Er wählt das Buch in einer Stunde.

Er wählt pro Stunde ein Buch.

$40 \mathrm{a}$ *Hän omistaa taloa vuoden.

40b Hän omistaa talon vuoden. Er besitzt das Haus ein Jahr.

$40 \mathrm{c}$ ?Hän omistaa talon vuodessa. Er besitzt ein Haus pro Jahr.

4 la *Hän huomaa autoa hetkisen.

4lb ?Hän ehtii huomata autoa Er hat wenig Zeit, das Auto zu bemerken. hetkisen.

41c Hän huomaa auton hetkessä. Er bemerkt das Auto sofort. 
Die oben eingeführten Kategorien besitzen insofern syntaktische Realität, als die Beispiele voneinander abweichen. Hier sind nur einige Beobachtungen möglich:

- Die Kategorie Partitiv-Akkusativ scheint die einzige zu sein, die beide Zeitangaben ohne weiteres zuläßt. Diese Beobachtung dürfte die Annahme bestätigen, daß die Verba dieser Kategorie semantisch offen sind und nach zwei Aspekten hin interpretiert werden können.

- Kategorie I schließt den Akkusativ aus; dies wird auch in diesem Falle bestätigt.

- Kategorie Ila (partitivpetal) läßt den Akkusativ zu, unter den gleichen Bedingungen wie oben erwähnt.

- Kategorie IIc (akkusativpetal) verhält sich am unregelmäßigsten. Mir scheint, daß der semantische Aufbau des Verbs eine entscheidende Rolle spielt, und daß die Verba dieser Kategorie eine größere Verschiedenheit im Aufbau aufweisen als die Verba anderer Kategorien. Selbst das allgemeine Prinzip, daß dieselbe Kasusendung nicht wiederholt werden darf, z.B. * Hän lukee kirjan tunnin (vgl. Hakulinen - Karlsson 1979:179), scheint hier außer Kraft zu treten (Beispiel 40b). Dies Beispiel wirkt zwar fremd (Hän omistaa talon vain vuoden 'nur ein Jahr' klingt natürlicher), aber "grammatikalisch". Bisweilen (Beispiel 39a) scheint das semantische Feld des Verbs sich zu einem neuen Aspekt zu dehnen (vgl. die deutsche Übersetzung). Beim Verb huomata ist das nicht möglich, und der Partitiv wird abgelehnt (41a). Das Beispiel (4lb) wirft die Frage auf, warum der Partitiv in diesem Zusammenhang toleriert wird. Mir scheint, daß es durch eine "Segmentierung" kaum zu erklären wäre. Es würde eher die Modalität sein: ehtiä bedeutet 'in Gelegenheit sein, über 'Zeit' zu verfügen; Zeit haben' und der Partitiv macht die Aussage negativ ("kaum Zeit; nur wenig Zeit"). Im oben erwähnten Beispiel (32b) dürfte diese Erklärung ebenfalls gültig sein. Eine theoretisch interessante Konsequenz ergibt sich aus der Beobachtung, daß huomata (Satz $4 \mathrm{lb}$ ), falls es akzeptiert wird, auch anfängt, sich semantisch auszudehnen. Es scheint aber normaliter ein Verb zu sein, das eine temporale Bestimmung nicht zuläßt. Das scheint eine Indikation dafür zu sein, daß die akkusativpetalen Verba sich semantisch nicht auf eine "Bewegung" beziehen: die räumliche Bezogenheit ist ja ohne weiteres in eine temporale Dimension zu transponieren. 
4. Einige Schlußfolgerungen

4.0. Die obigen Ausführungen dürften gezeigt haben, daß die Kategorie 'Objekt' im Finnischen ebenso kompliziert ist wie die Kategorie 'Subjekt' (dazu z.B. Auli Hakulinen 1982, Pentti Leino 1982). Die Zweiteilung, wie sie hier vorgeschlagen wird, hätte jedenfalls den Vorteil, daß auch die Probleme geteilt werden könnten. Ich bin hier grammatikalischen Problemen wie der Erörterung der grammatikalischen Etiketten aus dem Weg gegangen, etwa der Frage, was nun 'Partitivadverbial' und was 'Objekt' sei. Wenn auch Probleme geblieben sind, so hat sich doch ihre Gestalt verändert. Wenn man 'Irresultativität' und 'Resultativität' oder 'Unmarkiertheit' und 'Markiertheit' in 'Partitivpetalität' und 'Akkusativpetalität' verändert, kann man jedenfalls neue Fragen stellen und auf unerwartete Tatbestände stoßen. Ich werde kurz noch ein paar Fragen berühren, die mit meinem Ausgangspunkt, dem Unterricht für nicht-finnische Erlerner, zusammenhängen.

4.1. Die letzte Etappe der Objektdiskussion ist von Auli Hakulinen und Fred Karlsson initiiert durch die Feststellung, daß der Partitiv der üblichste Objektkasus sei (1979:181-182). Die obigen Ausführungen haben gezeigt, daß Faktoren mitspielen, die man bei Berechnungen und in der Analyse eigentlich nicht ausschließen sollte.

1) Es gibt Verba, die nur den Partitiv regieren.

2) Der Partitiv bezeichnet eine Unbestimmtheit des Objekts, die ich kurzweg 'unbestimmte Menge' (Stoffnamen Sg.) und 'indefinite Anzahl' (Plural) nenne.

3) Alle Verba regieren den Partitiv, wenn die Aussage verneinend ist oder als unsicher präsentiert wird.

(Vgl. Hakulinen - Karlsson 1979:182 über Gründe für den Partitiv)

Es wäre interessant zu erfahren, wie die Kasusdistribution sich verhält, wenn die obigen Fälle ausgeklammert werden, wo der Partitiv nicht nur als "Objekt" gewählt wird. Vor allem aus der Perspektive des Unterrichts wäre eine solche Analyse nützlich, bevor die Erlerner den "unmarkierten" Kasus mit dem "üblichsten Kasus" gleichsetzen und den Partitiv überall anwenden. Ich halte es nämlich durchaus für möglich, daß der Anteil des Akkusativs wächst, wenn man den Gebrauch mit dem entscheidenden Punkt, einem 
Nicht-Stoffnamen im Singular, in Beziehung bringt. Obwohl die Anzahl der partitivpetalen Verba an sich höher zu sein scheint als die der akkusativpetalen, sind viele Verba mit hoher Frequenz akkusativpetal.

Im Unterricht könnte von Nutzen sein, wenn die "automatische" Kasusdistribution (hier Kategorie I) aus den Objektregeln ausgeklammert würde; diese Kategorie könnte man lexikalisch erlernen, so wie z.B. Verba, die den Illativ regieren. In dieser und der partitivpetalen Kategorie ist der Partitiv der "syntaktische" Objektkasus, in Fällen, wo er in Opposition zum Akkusativ steht, ein "semantischer" Kasus. Es ist die Eigenartigkeit des fi. Systems, daß der Akkusativ ein "semantischer" Kasus ist. Mir scheint aber, daß diese Bezeichnungen eher Verwirrung stiften als das fi. System "erklären".

4.2 Eine der Schwierigkeiten vom Standpunkt des Erlerners ist die Flexibilität und Verschwommenheit der Grenzen zwischen den Kategorien. Scharf akzentuiert sind die Gegenpole, die kleine Gruppe Verba, die nur den Partitiv regieren, und eine ebenso geringe Gruppe, die stark akkusativpetal ist. Der Rest schwankt dazwischen, und die Grenzfälle zwischen den Gruppen sind auch für einen $\mathrm{fi}$. Sprecher arbiträr. Ich meine aber, daß die Kriterien für die Kategorien ausgearbeitet werden können, z.B. mit Hilfe systematisch zusammengestellter Testsätze, die die syntaktischen Reaktionen auf verschiedene ausgewählte Situationen und Aspekte beschreiben.

4.3. Aus der hier erörterten Perspektive scheinen einige Tendenzen im Sprachgebrauch eine Erklärung zu finden, vor allem die Tendenz zur Partitivisierung. Nehmen wir als Beispiel das Verb huomata, das, wie erwähnt, stark akkusativpetal ist. Es läßt daher den Partitiv nicht zu; dieser aber, wie oben illustriert wurde, trägt Bedeutungen, die frequent vonnöten sind. Es ist kein Zufall, daß es ein Verb gibt, das hartnäckig das gleiche semantische Feld zu betreten versucht, nämlich huomioida 'berücksichtigen; observieren; beobachten; Beobachtungen machen; bemerken' (vgl. den Artikel in Nykysuomen Sanakirja und Jouko Vesikansa 1976). Es zeigt also eine Tendenz, das von dem Stamm huomio 'Aufmerksamkeit; Observation, Beobachtung; Folgerung, Meinung' bestrittene Feld verbal auszudrücken; es nützt dabei sowohl den Akkusativ als auch den Partitiv aus. Eben diese Toleranz gegenüber dem Partitiv dürfte den Gebrauch des Verbs stimulieren, 
gegen alle Empfehlungen der Sprachpflege: mitunter braucht man den Partitiv eben.

PAIVI SCHOT-SAIKKU

\section{LITERATUR}

Fromm 1982 = Hans Fromm, Finnische Grammatik, Heidelberg 1982.

De Groot - Tommola 1984 = Caspar de Groot - Hannu Tommola (eds.), Aspect Bound. A foyage into the realm of Germanic, Slavonic and Finno-Ugrian Apectology. Dordrecht 1984.

Hakulinen - Karlsson = Auli Hakulinen - Fred Karlsson, Nykysuomen lauseoppia. Suomalaisen Kirjallisuuden Seuran Toimituksia 350.

Hakulinen 1982 = Auli Hakulinen, Subjektikategoria vai nominaalijäsenten subjektimaisuus. Suomen kielitieteellisen yhdistyksen julkaisuja 9: Lauseen jäsennyksen perusteet. Ss. 17 - 33. Turku 1982.

Heinämäki 1984 = Orvokki Heinämäki, Aspect in Finnisch. In: De Groot Tommola S. 153 - 177.

Itkonen 1974 (1976) = Terho Itkonen, Erään sijamuodon ongelmia. Separatum ex: Suomalainen Tiedeakatemia: esitelmät ja pöytäkirjat 1974 (1986), 173 - 217. Opuscula Instituti Linguae Fennicae Universitas Helsingiensis 53.

Kangasmaa-Minn 1984 = Eeva Kangasmaa-Minn, Tense, aspect and Aktionsart in Finno-Ugrian. In: De Groot - Tommola 1984 Ss. 77 - 93.

Leino 1982 = Pentti Leino, Miten suhtautua subjektiin? Suomen kielitieteellisen yhdistyksen julkaisuja 9: Lauseenjäsennyksen perusteet. Ss. 5 - 15. Turku 1982.

Leiwo 1983 = Matti Leiwo, On grammatical intuitions. Papers from the 7 th Scandinavian Conference of Linguistics. Vol. 1 - 2. Ed. by Fred Karlsson. Publications No. 10, 1983. University of Helsinki. Departement of General Linguistics. Ss. 622 - 633.

Lyons 1977 (Reprinted 1981) = John Lyons, Semantics. Vol. 1 - 2. Cambridge.

Siro 1956 = Paavo Siro, Die finnischen Lokalkasus als Bestimmungen des Verbums. Turun Yliopiston julkaisuja B: 57. Turku. 
Wande 1985 = Erling Wande, Sprachkontakt Finnisch-Ugrisch/-Schwedisch im heutigen Schweden: strukturelle Tendenzen. Vortrag gehalten auf dem 6. Finnougristenkongress in Syktyvkar am 29. Juli 1985.

Vesikansa 1976 = Jouko Vesikansa, Täsmennyssanasto. Nykysuomen oppaita nr. 1 Porvoo 1976. 